\title{
Prostatic Artery Embolization as a Primary Treatment for Benign Prostatic Hyperplasia: Preliminary Results in Two Patients
}

\author{
Francisco Cesar Carnevale - Alberto Azoubel Antunes • Joaquim Mauricio da Motta Leal Filho • \\ Luciana Mendes de Oliveira Cerri • Ronaldo Hueb Baroni · Antonio Sergio Zafred Marcelino • \\ Geraldo Campos Freire • Airton Mota Moreira • Miguel Srougi • Giovanni Guido Cerri
}

Received: 27 July 2009/Accepted: 23 September 2009/Published online: 12 November 2009

(C) Springer Science+Business Media, LLC and the Cardiovascular and Interventional Radiological Society of Europe (CIRSE) 2009

\begin{abstract}
Symptomatic benign prostatic hyperplasia (BPH) typically occurs in the sixth and seventh decades, and the most frequent obstructive urinary symptoms are hesitancy, decreased urinary stream, sensation of incomplete emptying, nocturia, frequency, and urgency. Various medications, specifically $5-\alpha$-reductase inhibitors and selective $\alpha$ blockers, can decrease the severity of the symptoms secondary to BPH, but prostatectomy is still considered to be the traditional method of management. We report the preliminary results for two patients with acute urinary retention due to $\mathrm{BPH}$, successfully treated by prostate artery
\end{abstract}

F. C. Carnevale · J. M. da Motta Leal Filho - A. M. Moreira Interventional Radiology Unit, Radiology Institute, Hospital das Clinicas, University of Sao Paulo Medical School, Av. Dr. Enéas de Carvalho Aguiar, 255, Sao Paulo, SP 05403-001, Brazil

A. A. Antunes - G. C. Freire - M. Srougi

Urology Department, Hospital das Clinicas, University of Sao

Paulo Medical School, Av. Dr. Enéas de Carvalho Aguiar, 255,

Sao Paulo, SP 05403-001, Brazil

L. M. de Oliveira Cerri · A. S. Z. Marcelino

Ultrasound Unit, Radiology Institute, Hospital das Clinicas,

University of Sao Paulo Medical School, Av. Dr. Enéas de

Carvalho Aguiar, 255, Sao Paulo, SP 05403-001, Brazil

R. H. Baroni

Magnetic Resonance Unit, Radiology Institute, Hospital das Clinicas, University of Sao Paulo Medical School, Av. Dr. Enéas de Carvalho Aguiar, 255, Sao Paulo, SP 05403-001, Brazil

G. G. Cerri

Radiology Department, Radiology Institute, Hospital das Clinicas, University of Sao Paulo Medical School, Av. Dr. Enéas de Carvalho Aguiar, 255, Sao Paulo, SP 05403-001, Brazil

F. C. Carnevale $(\square)$

Rua Teodoro Sampaio, 352/17, Sao Paulo, SP 05406-000, Brazil

e-mail: fcarnevale@uol.com.br embolization (PAE). The patients were investigated using the International Prostate Symptom Score, by digital rectal examination, urodynamic testing, prostate biopsy, transrectal ultrasound (US), and magnetic resonance imaging (MRI). Uroflowmetry and postvoid residual urine volume complemented the investigation at 30,90, and 180 days after PAE. The procedure was performed under local anesthesia; embolization of the prostate arteries was performed with a microcatheter and 300- to $500-\mu \mathrm{m}$ microspheres using complete stasis as the end point. One patient was subjected to bilateral PAE and the other to unilateral PAE; they urinated spontaneously after removal of the urethral catheter, 15 and 10 days after the procedure, respectively. At 6-month follow-up, US and MRI revealed a prostate reduction of $39.7 \%$ and $47.8 \%$, respectively, for the bilateral PAE and 25.5 and $27.8 \%$, respectively, for the patient submitted to unilateral PAE. The early results, at 6-month follow-up, for the two patients with BPH show a promising potential alternative for treatment with PAE.

Keywords Benign prostatic hyperplasia - Prostate · Embolization - Urinary retention · Prostatic artery

\section{Introduction}

Symptomatic benign prostatic hyperplasia (BPH) typically occurs in the sixth and seventh decades, and more than $40 \%$ of men older than this present with clinical manifestations of this tumor [1-4]. The most frequent obstructive urinary symptoms are hesitancy, decreased urinary stream, intermittency, sensation of incomplete emptying, nocturia, frequency, and urgency [2]. The standard management of BPH depends on the overall health of the patient and the severity of the symptoms. Voiding difficulties attributable to BPH 
can be quantified with the International Prostatic Symptom Score. Various medications, specifically 5 - $\alpha$-reductase inhibitors and selective $\alpha$-blockers, can decrease the severity of voiding symptoms secondary to BPH [1, 4]. Minimally invasive techniques have been developed as alternatives for treatment of $\mathrm{BPH}$, such as transurethral microwave thermotherapy and other laser ablations, but prostatectomy (accomplished by transurethral or open surgical means) constitutes the traditional surgical treatment for BPH [5-8]. Taking into account the comorbidities, surgical intervention in this age group is considered to be high-risk. Prostate artery embolization (PAE) has been used mainly to control massive hemorrhage after prostatectomy or prostate biopsy [9-13]. DeMeritt et al. [14] described a patient with BPH who had severe gross hematuria and was subjected to PAE with polyvinyl alcohol particles. The patient stopped bleeding immediately after embolization and the size of the prostate reduced to $52 \%$ and $62 \%$ of the initial size by the 5 - and $12-$ month follow-up, respectively. Recently, an experimental study of PAE in pigs showed a significant reduction in prostate volume after embolization without compromising the sexual function and erectile function of the animals [15]. We report the preliminary results of PAE with microspheres as a primary treatment in two patients with acute urinary retention due to BPH initially managed by selective $\alpha$ blockers and urethral catheters.

\section{Materials and Methods}

Two patients with acute urinary retention due to BPH had been managed by selective $\alpha$-blockers and urethral catheters and were waiting for surgery. Both patients were treated previously with selective $\alpha$-blockers without success and stopped taking medication 1 month before PAE. Before intervention, the patients were investigated using the International Prostate Symptom Score, by digital rectal examination, urodynamic testing, prostate biopsy, transrectal ultrasound (US), and magnetic resonance imaging (MRI). Malignancy and any other cause of voiding dysfunction that could be correlated with the urinary obstruction were excluded. The Ethical Committee approved the study and the patients signed an informed consent for PAE as an alternative of treatment. After PAE, uroflowmetry, postvoid residual urine volume, US, and MRI were used to complement investigation at 30,90, and 180 days after PAE. The interval between contrast-enhanced US and MRI was less than 3 days for both patients.

\section{Ultrasound}

Prostate volume and intravesical prostatic protrusion (IPP) were measured by standard transrectal ultrasound using
ApplioTM equipment (Toshiba America Medical Systems). Prostate vascularization was examined with Doppler and after the microbubble product of the sonicated contrast agent Definity (Bristol Meyers Squibb, Billerica, MA, USA) was injected in bolus through the antecubital vein in a dose of $1 \mathrm{ml}$, followed by $10 \mathrm{ml}$ of saline solution. After $\mathrm{PAE}$, the residual urine volume was assessed by the transabdominal approach.

\section{Magnetic Resonance Imaging}

The imaging protocol for all examinations was the same: on a 1.5-T magnet using a phased-array, 12-channel body coil (GE Healthcare, Milwaukee, WI, USA). Because of technical issues, we used fat suppression for pre-embolization studies but not for postembolization ones. Additionally, we performed two axial sequences for the study of the pelvis. Gadolinium chelate (Magnevist; Bayer-Schering, Germany) was injected through an antecubital vein in a dose of $0.2 \mathrm{ml} /$ $\mathrm{kg}$, at $2 \mathrm{ml} / \mathrm{s}$, using an automated bolus injection system. Prostate measurements and volume were evaluated.

\section{Prostate Artery Embolization}

Patients were referred for angiography and embolization in the interventional radiology suite using an Allura DSA unit (Philips, the Netherlands) and with nonionic Visipaque contrast medium (Iodixanol, $320 \mathrm{mg} \mathrm{I} / \mathrm{ml}$; GE, Healthcare, Europe). A prophylactic single dose of $200 \mathrm{mg}$ ciprofloxacin was given prior to the procedure. Intervention was performed under local anesthesia through the right transfemoral approach. Initial pelvic angiography was performed to evaluate the iliac vessels and prostate arteries during the arterial and late phases. Selective digital subtraction angiography of the right and left internal iliac arteries was performed using a 5-Fr Cobra 2 catheter to better assess the blood supply to the prostate. Superselective catheterization of the right and left inferior vesicle arteries was then performed using a microcatheter (Embocath; Biosphere Medical, Rockland, ME, USA) and angiography was performed by manually injecting $3-5 \mathrm{ml}$ of contrast medium to ensure that the tip of the microcatheter was inside or at the ostium of the prostatic arteries. Microspheres (Embosphere; Biosphere Medical) calibrated to $300-500 \mu \mathrm{m}$ in diameter were used for embolization. Each 2-ml vial of microspheres was diluted in a mixture of $20 \mathrm{ml}$ of $50 \%$ iodinated contrast medium plus $50 \%$ normal saline solution. The mixture was slowly injected under fluoroscopic guidance. Embolization of the prostate arteries arising from the inferior vesical arteries was performed to stasis without reflux of the mixture to undesired arteries. Follow-up angiography was performed manually with the microcatheter at the inferior vesical artery and using the 
pump with the 5-Fr catheter at the anterior branch of the internal iliac artery checking any further blood supply to the prostate. Embolization was then performed on the other side using the same technique.

\section{Patient 1}

A 67-year-old man with arterial hypertension and acute urinary retention due to BPH was admitted to the urology service, where he was treated with transurethral catheter drainage. Digital rectal examination showed a prostate compatible with $50 \mathrm{~g}$. The prostate specific antigen (PSA) level was $12.9 \mathrm{ng} / \mathrm{ml}$ (reference range: $0-4 \mathrm{ng} / \mathrm{ml}$ ). Transrectal US showed an enlarged heterogeneous prostate, $63 \mathrm{~g}$ in volume, and an IPP of $9 \mathrm{~mm}$. Prostate biopsy (12 samples) revealed mild nonspecific chronic prostatitis and acute prostatitis. MRI revealed an enlarged prostate, $69 \mathrm{~g}$ in volume, with heterogeneous high-signal intensity nodules of probable hyperplasia in the central gland, protruding at the bladder neck. In the PAE procedure, bilateral prostate artery embolization was performed successfully, without complications. Procedure/fluoroscopy time was $160 / 59 \mathrm{~min}$, respectively.

\section{Patient 2}

A 68-year-old man with atrial flutter and arterial hypertension, who developed acute urinary retention due to $\mathrm{BPH}$, was admitted to the urology service and treated with transurethral catheter drainage. Digital rectal examination showed a prostate compatible with $50 \mathrm{~g}$. The PSA level was $7.1 \mathrm{ng} / \mathrm{ml}$ (reference range: $0-4 \mathrm{ng} / \mathrm{ml}$ ). Transrectal US showed an enlarged heterogeneous prostate, $51 \mathrm{~g}$ in volume, with asymmetric hypertrophy of the internal gland, mainly at the right lobe, and an IPP of $16 \mathrm{~mm}$. Prostate biopsy (12 samples) revealed severe chronic prostatitis. MRI showed an enlarged prostate, $54 \mathrm{~g}$ in volume, with a heterogeneous high-signal intensity due to probable hyperplasia in the central gland, with a more discrete avascular cystic nodule of $1.1 \mathrm{~cm}$ on the right protruding at the bladder neck. In the PAE procedure, unilateral prostate artery embolization was performed successfully without complications. Left prostate arteries were not visualized during angiography. Procedure/fluoroscopy time was $250 / 95 \mathrm{~min}$, respectively.

\section{Results}

The prostate volumes, IPP, and postvoid residual urine volume at 1-, 3-, and 6-month follow-up, measured by US and MRI, for both patients are reported in Tables 1 and 2.

\section{Patient 1 Post-PAE Follow-Up (Fig. 1)}

The patient complained of mild retropubic pain for $24 \mathrm{~h}$, which was treated with nonopioid analgesic. He had no fever or hematuria and was discharged 3 days after PAE. $\mathrm{He}$ urinated spontaneously upon urethral catheter removal 15 days after the procedure.
Table 1 Pre and post US and MRI evaluations for the patient subjected to bilateral prostate artery embolization (PAE; patient 1)

Table 2 Pre and post US and MRI evaluations for the patient subjected to unilateral prostate artery embolization (PAE; patient 2)

\begin{tabular}{lllll}
\hline & Pre PAE & $\begin{array}{l}\text { 1-month } \\
\text { follow-up }\end{array}$ & $\begin{array}{l}\text { 3-month } \\
\text { follow-up }\end{array}$ & $\begin{array}{l}\text { 6-month } \\
\text { follow-up }\end{array}$ \\
\hline MRI Prostate volume (g) & 69 & 42 & 38 & 36 \\
Prostate volume reduction (\%) & - & 39.1 & 44.9 & 47.8 \\
US Prostate volume (g) & 63 & 42 & 38 & 38 \\
Prostate volume reduction (\%) & - & 33.3 & 39.7 & 39.7 \\
Intravesical prostatic protrusion (mm) & 9 & 4 & 4 & 5 \\
Postvoid residual urine volume (ml) & With transurethral & 8 & 5 & Absent \\
& catheter & & & \\
\hline
\end{tabular}

\begin{tabular}{lllll}
\hline & Pre PAE & $\begin{array}{l}\text { 1-month } \\
\text { follow-up }\end{array}$ & $\begin{array}{l}\text { 3-month } \\
\text { follow-up }\end{array}$ & $\begin{array}{l}\text { 6-month } \\
\text { follow-up }\end{array}$ \\
\hline MRI Prostate volume (g) & 54 & 41 & 39 & 39 \\
Prostate volume reduction (\%) & - & 24.1 & 27.8 & 27.8 \\
US Prostate volume (g) & 51 & 41 & 38 & 38 \\
Prostate volume reduction (\%) & - & 19.6 & 25.5 & 25.5 \\
Intravesical prostatic protrusion (mm) & 16 & 16 & 16 & 15 \\
Postvoid residual urine volume (ml) & With transurethral & 110 & 68 & 85 \\
& catheter & & & \\
\hline
\end{tabular}



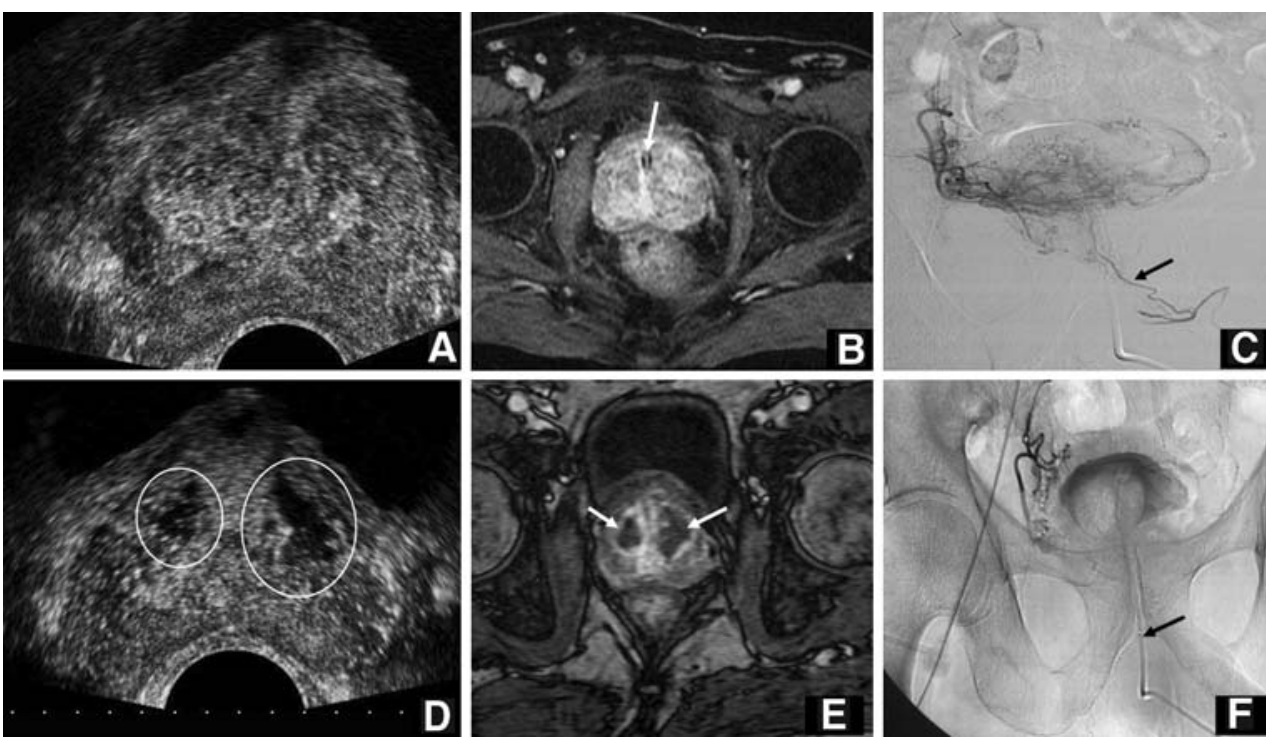

Fig. 1 Patient 1. A Transversal view of the prostate gland. Baseline pre-embolization contrast-enhanced ultrasound. Homogeneous enhancement of the internal gland. B Pre-embolization MRI. Axial postcontrast T1-weighted MRI depicting the enlarged prostate due to central gland nodules. Note the presence of the urethral catheter (white arrow). C Angiogram pre-embolization showing the right inferior vesical artery and the right prostate branches. The branches to the prostate communicate with the corresponding vessels of the opposite

\section{One-Month Follow-Up}

The urine jet was increased; postvoid residual urine volume was $8 \mathrm{ml}$ and IPP $4 \mathrm{~mm}$. There was a prostate volume reduction of $33.3 \%$ by US and $39.1 \%$ by MRI.

\section{Three-Month Follow-Up}

The patient was voiding normally; postvoid residual urine volume was $5 \mathrm{ml}$ and IPP $4 \mathrm{~mm}$. There was a prostate volume reduction of $39.7 \%$ by US and $44.9 \%$ by MRI.

\section{Six-Month Follow-Up}

The patient was voiding normally; postvoid residual urine was absent and the IPP $5 \mathrm{~mm}$. The prostate volume reduction was $39.7 \%$ by US and $47.8 \%$ by MRI.

\section{Patient 2 Post-PAE Follow-Up (Fig. 2)}

The patient was asymptomatic and discharged 3 days after PAE. He urinated spontaneously after urethral catheter removal 10 days after the procedure.

\section{One-Month Follow-Up}

There were episodes of nocturia and increase in the urine jet; postvoid residual urine volume was $110 \mathrm{ml}$ and IPP side (black arrow). D Six-month follow-up after PAE contrastenhanced ultrasound. Normal enhancement in the hyperechoic areas of internal gland and hypoechoic avascular areas (white circles). E Sixmonth postembolization MRI. Axial postcontrast T1-weighted MRI depicting bilateral avascular areas in the central gland (white arrows) and a reduction in prostate size. F Angiogram after right inferior vesical artery and right prostate branch embolization. Transurethral catheter in place (black arrow)

$16 \mathrm{~mm}$ (stable). The prostate volume reduction was $19.6 \%$ by US and $24.1 \%$ by MRI. MRI depicted an increase in size of the previously seen avascular nodule on the right central gland (from 1.1 to $1.3 \mathrm{~cm}$ ).

\section{Three-Month Follow-Up}

The patient was voiding normally; postvoid residual urine volume was $68 \mathrm{ml}$ and IPP $16 \mathrm{~mm}$. There was a prostate volume reduction of $25.5 \%$ by US and $27.8 \%$ by MRI. MRI showed persistence of the avascular area in the right central gland.

\section{Six-Month Follow-Up}

The patient was voiding normally; postvoid residual urine volume was $85 \mathrm{ml}$ and IPP $15 \mathrm{~mm}$. US and MRI showed prostate volume stability in comparison to the last controls.

\section{Discussion}

BPH refers to the increase in size of the prostate in middleaged and elderly men [1]. The normal prostate is composed of a combination of glandular, stromal, and smooth muscle cells. BPH is due to a proliferation of glandular elements, fibromuscular (stromal) elements, or both, resulting in the formation of large, fairly discrete nodules in the 

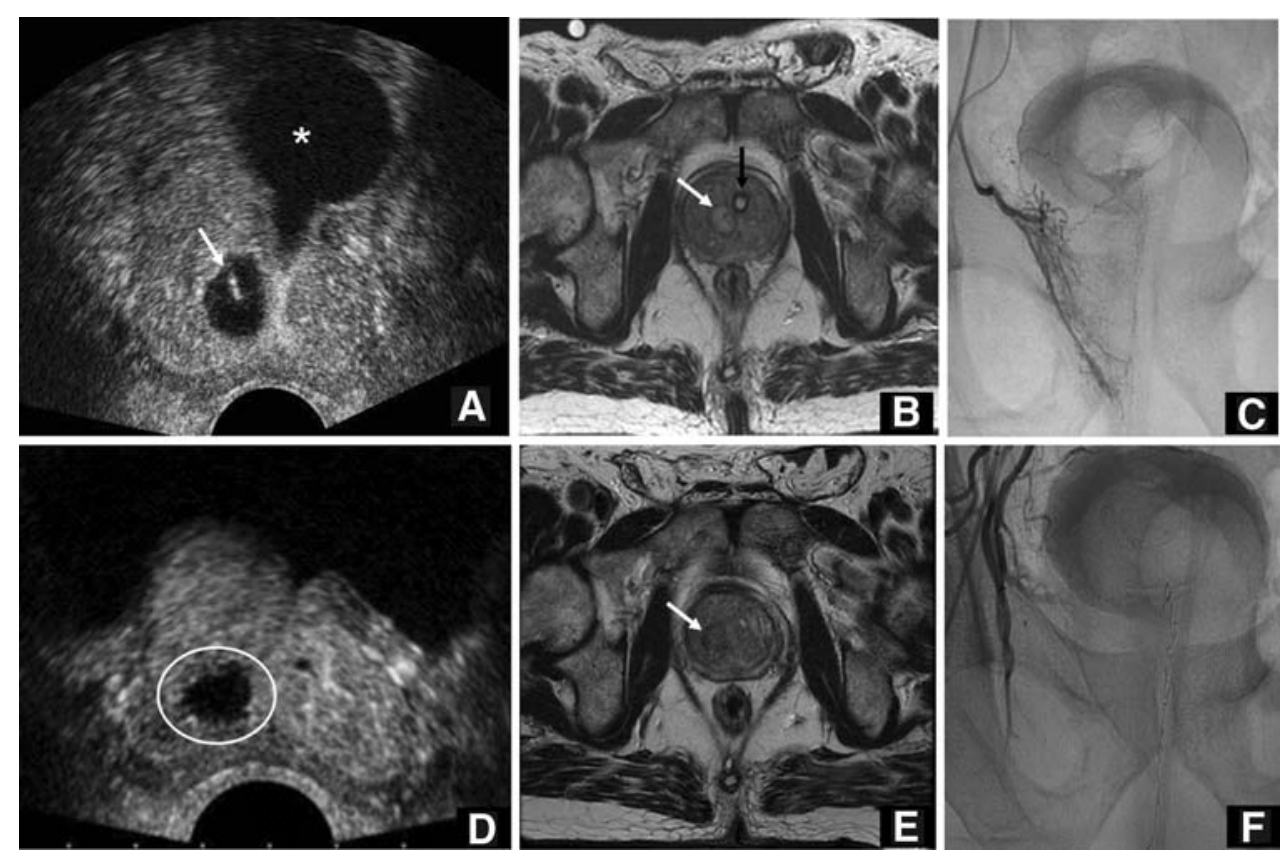

Fig. 2 Patient 2. A Baseline pre-embolization contrast-enhanced ultrasound. Tranverse view of the prostate gland. Asymmetric enlargement of the internal gland. Homogeneous enhancement of the internal gland characterized by hyperechoic areas. Note the small cyst in the right internal gland (white arrow) and the Foley catheter (*). B Pre-embolization MRI. Axial T2-weighted MRI depicting an enlarged prostate due to nodules in the central gland, with a more cystic nodule on the right side (white arrow). Note the presence of the urethral catheter (black arrow). C Angiogram pre-embolization

periurethral region of the prostate $[16,17]$. Symptomatic BPH typically occurs in the sixth and seventh decades, and more than $40 \%$ of men older than this present with clinical manifestations of this tumor [2,4]. The most frequent obstructive urinary symptoms are hesitancy, decreased urinary stream, intermittency, sensation of incomplete emptying, nocturia, frequency, and urgency, which severely affect the patients' quality of life. The standard management of BPH is based on the overall health of the patient and the severity of symptoms $[1,2,4]$. Medications, specifically 5 - $\alpha$-reductase inhibitors and selective $\alpha$ blockers, can decrease the severity of voiding symptoms secondary to $\mathrm{BPH}$, and minimally invasive techniques such as transurethral microwave thermotherapy, interstitial laser thermoablation, transurethral needle ablation, and waterinduced thermotherapy have been indicated to treat complications of BPH [2, 4-8]. Even with the development of new therapies, prostatectomy accomplished by transurethral or open surgical means constitutes the traditional surgical treatment for $\mathrm{BPH}$ [4], but considering the comorbidities, prostatectomy in this age group is considered to be high-risk $[1,4]$.

Some reports show that PAE has been used successfully, mainly to control massive hemorrhage after prostatectomy showing the right inferior vesical artery and the right prostate branches delimiting the right side of the gland. D Six-month followup after PAE. Contrast-enhanced ultrasound shows normal enhancement in the hyperechoic areas of the internal gland and hypoechoic avascular area (white circle). E Six-month postembolization MRI. Axial T2-weighted MRI depicting a reduction in prostate size and the persistence of a conspicuous cystic nodule on the right side (white arrow). F Angiogram control after right inferior vesical artery and right prostate branch embolization

or prostate biopsy [9-13]. DeMeritt et al. [14] described a patient with BPH who had severe gross hematuria and was subjected to PAE with polyvinyl alcohol particles. The patient stopped bleeding immediately after embolization and had improved urinary symptoms, accompanied by a dramatic reduction in prostate volume $52 \%$ and $62 \%$ of the initial size by the 5- and 12-month follow-up, respectively). Recently, an experimental study of PAE in pigs showed that PAE could be performed safely, with a significant reduction of prostate volume, without compromising the sexual function and erectile function of the animals [15]. Transarterial embolization has been widely used for treatment of symptomatic uterine fibroids, with the aim of reducing the fibroids and uterus, with satisfactory clinical outcomes [18-22]. One of the aims of fibroid embolization is that ischemia or hypoxia induces apoptosis, necrosis, and sclerosis in, and subsequent shrinkage of, tumors $[18,23,24]$. The blood supply to the prostate is from the anterior branch of the internal iliac artery, mainly by the inferior vesicle artery, which subsequently branches into the urethral and capsular vessels. Minor prostatic vessels also arise from the internal pudendal and middle hemorrhoidal arteries. Due to the similarity of the cells and arterial vascular anatomy observed in the prostate, PAE 
could be used as an alternative treatment, with the aim of reducing the prostate size and producing relief from symptoms caused by BPH.

One of the concerns with PAE is related to the risk of bladder wall ischemia and necrosis after inferior vesical artery embolization. The most important blood supply to the bladder is from the inferior vesicle, superior vesicle, and a vesical branch of the obturator artery. Bladder necrosis is a rare entity in the urological literature. Most such complications were secondary to pelvic artery embolization to control intractable hemorrhage from a pelvic fracture without the use of superselective catheterization using the microcatheter coaxial system $[25,26]$. Recently, in eight patients with a history of refractory hematuria of prostatic origin, no cases of bladder necrosis related to selective arterial prostatic embolization were observed [13].

In our initial study, two patients treated by PAE had acute urinary retention due to $\mathrm{BPH}$. The procedure was performed under local anesthesia using the same standard technique as for fibroid embolization, and only the prostate arteries arising from the inferior vesical arteries were embolized. One patient complained of mild retropubic pain for $24 \mathrm{~h}$, which was treated with nonopioid analgesic, and was discharged 3 days after PAE. But we think he could have been discharged on the day of the procedure.

The main differences between PAE and fibroid embolization are related to dealing with thin tortuous atherosclerotic vessels, difficulty of visualization, and superselective catheterization of the inferior vesical artery and prostate arteries. It is much more difficult than performing a fibroid embolization and requires the use of microcatheters in all cases. Our procedure was prolonged, mainly for the unilateral PAE. The learning curve, the prolonged time required to identify and catheterize the prostate arteries, and the absence of the left prostate arteries in the second patient were the main reasons for the long procedures. Both urethral catheters were removed during the second-week follow-up and patients urinated spontaneously. The urine jet increased with time, with reduction of the postvoid residual urine volume. Imaging follow-up with US and MRI showed the most important prostate shrinkage during the first month after PAE for both patients, and prostate reduction was still observed at the last 6-month control. The patient who had bilateral PAE had a prostate reduction of $33.3 \%$ by US and $39.1 \%$ by MRI at the 30-day examination and $39.7 \%$ by US and $47.8 \%$ by MRI at the 6-month control. The patient who had unilateral PAE had a prostate reduction of $19.6 \%$ by US and $24.1 \%$ by MRI at the 30-day examination and $25.5 \%$ by US and $27.8 \%$ by MRI at the 6-month control.

The early results, at 6-month follow-up, of the two patients with BPH show a promising potential alternative treatment in PAE. Future studies with long-term follow-up, unilateral or bilateral PAE, and different-sized microspheres, and a larger study to validate our observations, are required to answer some questions.

\section{References}

1. Emberton M, Andriole GL, de la Rosette J et al (2003) Benign prostatic hyperplasia. A progressive disease of aging men. Urology 61:267-273

2. Ziada A, Rosenblum M, Crawford ED (1999) Benign prostatic hyperplasia: an overview. Urology 53(3; Suppl 3a):1-6

3. Guess HA, Arrighi HM, Metter EJ et al (1990) Cumulative prevalence of prostatism matches the autopsy prevalence of benign prostatic hyperplasia. Prostate 17(3):241-246

4. AUA Practice Guidelines Committee (2003) AUA guideline on management of benign prostatic hyperplasia. I. Diagnosis and treatment recommendations. J Urol 170(2; pt 1):530-547

5. Roehrborn CG, Preminger G, Newhall P et al (1998) Microwave thermotherapy for benign prostatic hyperplasia with the Dornier urowave: results of a randomized, double-blind, multicenter, sham-controlled trial. Urology 51(1):19-28

6. Wheelahan J, Scott NA, Cartmill R et al (2000) Minimally invasive laser techniques for prostatectomy: a systematic review. BJU Int 86(7):805-815

7. Roehrborn CG, Issa MM, Bruskewitz RC et al (1988) Transurethral needle ablation for benign prostatic hyperplasia: 12-month results of a prospective, multicenter U.S. study. Urology 51(3):415-421

8. Cioanta I, Muschter R (2000) Water-induced thermotherapy for benign prostatic hyperplasia. Tech Urol 6(4):294-299

9. Mitchell ME, Waltman AC, Athanasoulis CA et al (1976) Control of massive prostatic bleeding with angiographic techniques. $\mathrm{J}$ Urol 115:692-695

10. Appleton DS, Sibley GN, Doyle PT (1988) Internal iliac artery embolisation for the control of severe bladder and prostate haemorrhage. Br J Urol 61(1):45-47

11. Michel F, Dubruille T, Cercueil JP et al (2002) Arterial embolization for massive hematuria following transurethral prostatectomy. J Urol 168(6):2550-2551

12. Barbieri A, Simonazzi M, Marcato C et al (2002) Massive hematuria after transurethral resection of the prostate: management by intra-arterial embolization. Urol Int 69(4):318-320

13. Rastinehad AR, Caplin DM, Ost MC et al (2008) Selective arterial prostatic embolization (SAPE) for refractory hematuria of prostatic origin. Urology 71(2):181-184

14. DeMeritt JS, Elmasri FF, Esposito MP, Rosenberg GS (2000) Relief of benign prostatic hyperplasia-related bladder outlet obstruction after transarterial polyvinyl alcohol prostate embolization. J Vasc Interv Radiol 11(6):767-770

15. Sun F, Sánchez FM, Crisóstomo V et al (2008) Benign prostatic hyperplasia: transcatheter arterial embolization as potential treatment-preliminary study in pigs. Radiology 246(3):783-789

16. Caine M, Raz S, Zeigler M (1975) Adrenergic and cholinergic receptors in the human prostate, prostatic capsule and bladder neck. Br J Urol 47(2):193-202

17. Lepor H, Shapiro E (1984) Characterization of alpha1 adrenergic receptors in human benign prostatic hyperplasia. J Urol 132(6): 1226-1229

18. Pinto I, Chimeno P, Romo A et al (2003) Uterine fibroids: uterine artery embolization versus abdominal hysterectomy for treatment-a prospective, randomized, and controlled clinical trial. Radiology 226(2):425-431 
19. Mirsadraee S, Tuite D, Nicholson A (2008) Uterine artery embolization for ureteric obstruction secondary to fibroids. CardioVasc Interv Radiol 31(6): 1094-1099

20. Firouznia K, Ghanaati H, Sanaati M, Jalali AH, Shakiba M (2008) Uterine artery embolization in 101 cases of uterine fibroids: do size, location, and number of fibroids affect therapeutic success and complications? CardioVasc Interv Radiol 31(3): $521-526$

21. Hehenkamp WJ, Volkers NA, Bartholomeus W, de Blok S, Birnie E, Reekers JA, Ankum WM (2007) Sexuality and body image after uterine artery embolization and hysterectomy in the treatment of uterine fibroids: a randomized comparison. CardioVasc Interv Radiol 30(5):866-875

22. Dumousset E, Chabrot P, Rabischong B, Mazet N, Nasser S, Darcha C, Garcier JM, Mage G, Boyer L (2008) Preoperative uterine artery embolization (PUAE) before uterine fibroid myomectomy. CardioVasc Interv Radiol 31(3):514-520

23. Walker WJ, Pelage JP (2002) Uterine artery embolisation for symptomatic fibroids: clinical results in 400 women with imaging follow up. BJOG 109(11):1262-1272

24. Pelage JP, Le Dref O, Soyer P et al (2000) Fibroid-related menorrhagia: treatment with superselective embolization of the uterine arteries and midterm follow-up. Radiology 215(2):428431

25. Hietela SO (1978) Urinary bladder necrosis following selective embolization of the internal iliac artery. Acta Radiol Diagn (Stockh) 19(2):316-320

26. Sieber PR (1994) Bladder necrosis secondary to pelvic embolization: case report and literature review. J Urol 151(2):422 